\title{
MATERIAL SYMMETRY AND THE EVOLUTION OF ANISOTROPIES IN A SIMPLE MATERIAL-II. THE EVOLUTION OF MATERIAL SYMMETRY
}

\author{
Mehrdad Negahban* and Alan S. Wineman \\ Department of Mechanical Engineering and Applied Mechanics, The University of Michigan, \\ Ann Arbor, MI 48109 , U.S.A.
}

(Received 18 October 1988; in revised form 10 March 1989)

\begin{abstract}
A proper evolutionary definition of material symmetry is presented. The evolution of a material's symmetries as the material undergoes a deformation history is followed, and a procedure for the identification of those changes which are common to all simple materials is provided. Explicit results are presented for initially isotropic, transversely isotropic, and orthotropic materials undergoing simple deformation histories.
\end{abstract}

\section{INTRODUCTION}

In this paper, we will follow the evolution of a material's symmetries as the material undergoes a deformation history. The purpose is to identify those changes which are common to all simple materials.

Section 2 covers the preliminaries from continuum mechanics.

Sections 3 and 4 are devoted to explaining what is meant by material symmetry (MS) and material symmetry groups (MSGs). The MS is defined not as an absolute property of a material, but as a property associated with the state of the material. This definition allows MS to change as the material changes its state.

Section 5 states the problem and provides its solution. We show that the current MSG of the material is obtained as the superset of the intersection of the material's initial MSG with a group of transformations which describe the symmetries of the deformations the material has undergone.

Sections 6-10 confine their attention to materials which are initially in a state such that their MS can be associated with a group of orthogonal transformations (i.e. the material's group of MSGs can be generated from an orthogonal generator). In particular, explicit results are presented for initially isotropic, transversely isotropic, and orthotropic materials. In each case, several different deformation histories are considered.

There is a general lack of references on the subject of the evolution of anisotropies from a continuum point of view. The need for such a study has for some time been expressed in the field, and is described by J. L. Erickson [1] who stated: "For the most part, non-linear continuum theory has been based on the premise that the symmetry of a material never changes. To analyze common phase transition, we need to revise such a theory of symmetry, but this is easier said than done...".

\section{PRELIMINARY DEFINITIONS}

The reference configuration $\kappa$ is a configuration of the material body which is selected as a tool for labeling different material points in the body, and which is usually also used as a reference for the comparison of the different configurations taken by the material body during its motion (see Fig. 1). We note that the reference configuration need not ever be taken by the actual material body.

Let $r_{k}(X)$ denote the position of the material point (particle) $X$ in the reference configuration $\kappa$ and let $x\left[r_{x}(X), s\right]$ denote the position of $X$ in the configuration taken by the body at time $s$. The deformation gradient $F_{\alpha}$, which compares the configuration of the smallest neighborhood of $\mathrm{X}$ at time $s$ to its reference configuration, is defined to be $\mathrm{F}_{\alpha}\left[r_{\kappa}(X), s\right]$

* Present address: Department of Engineering Mechanics, University of Nebraska-Lincoln, Lincoln, NB 68588. 0347.

Contributed by K. R. Rajagopal. 


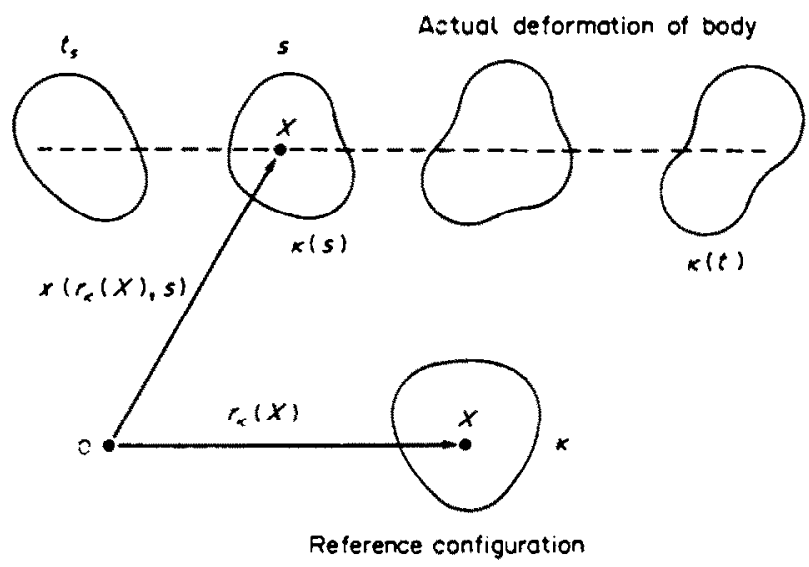

Fig. 1. Kinematical description of continuum bodies.

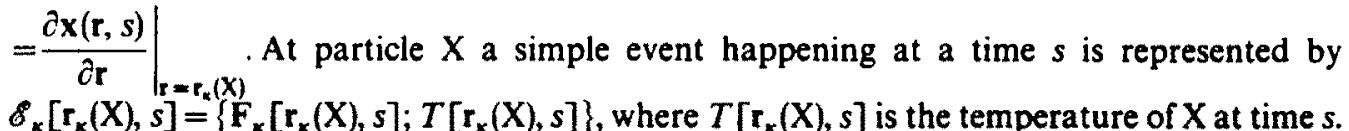
The history of simple events happening to $X$ starting at time $t_{1}$ and ending at time $t_{2}$ is denoted by $\mathscr{H}_{k}\left[r_{x}(\mathrm{X}), t_{1} \rightarrow t_{2}\right]$ and defined by

$$
\mathscr{H}_{\kappa}\left[\mathbf{r}_{\kappa}(\mathrm{X}) ; t_{1} \rightarrow t_{2}\right]=\left\{\mathscr{E} \mid \mathscr{E}=\mathscr{E}_{\kappa}\left[\mathbf{r}_{\kappa}(\mathrm{X}), \alpha\right] \text { for } \alpha \in\left(t_{1}, t_{2}\right]\right\} \text {. }
$$

The initial (virgin) time, $t_{v}\left[r_{x}(X)\right]$, represents the first instant at which the material point $\mathrm{X}$ is considered as a simple material. In other words, the "simple life" of a material starts at the initial time. The initial state of a material point is represented by $\mathscr{S}_{\mathrm{vk}}\left[\mathrm{r}_{\kappa}(\mathrm{X})\right]$ and contains all information pertaining to the influence of events before the initial time on the response of the material after this time.

The state of a simple material point at time $s$ is described by the union of the initial state and the history of simple events undergone by the material point from the initial time up to time $s^{\dagger}$ Letting $\mathscr{S}_{\kappa}\left[\mathrm{r}_{\kappa}(\mathrm{X}), s\right]$ represent the state of the material point $\mathrm{X}$ at time $s$ then

$$
\mathscr{S}_{\kappa}\left[\mathbf{r}_{\kappa}(\mathrm{X}), s\right]=\mathscr{S}_{v \kappa}\left[\mathbf{r}_{\kappa}(\mathrm{X})\right] \cup \mathscr{H}_{\kappa}\left[\mathbf{r}_{\kappa}(\mathrm{X}) ; t_{v} \rightarrow s\right] \text {. }
$$

\subsection{Stress in a simple material}

The constitutive equation for the stress response of a simple material is given by

$$
\sigma\left[\mathbf{r}_{\kappa}(\mathrm{X}), t\right]=\mathfrak{J}_{\kappa}\left\{\mathscr{S}_{\kappa}\left[\mathbf{r}_{\kappa}(\mathrm{X}), t\right]\right\} \text {, }
$$

where $\sigma\left[\boldsymbol{r}_{x}(X), t\right]$ is the Cauchy Stress: at the material point $X$ at time $t$, and $\mathfrak{J}_{k}$ is the constitutive (or response) functional which relates the state at the material point to the stress at that point.

The starting time, $t_{s}\left[r_{x}(X)\right]$, is selected as any convenient, but otherwise arbitrary, time equal or greater than the initial time. The explicit form of $\mathfrak{I}_{\kappa}$ will contain all necessary information of events happening before this time, and these events will be considered as part of the unchangeable past. However, to use the explicit form of $\mathfrak{I}_{x}$ in calculation, it is necessary to specify simple events occurring after this time. Therefore, the explicit form of $\mathfrak{I}_{\boldsymbol{\kappa}}$ provides the user with only the means to change the history of events beginning at the starting time. An example of a starting time, other than the initial time, is seen when a simple material which is a fluid at its initial time is solidified and a seemingly new constitutive equation is started at the point of solidification to describe the response of this material in its solid "state".

\footnotetext{
"In some uses of the term "state" there is no distinction made between "the state of a material", "the representation of the state of the material", and "equivalent states of the material". In our usage, we will consider two states of a material as equivalent if their representations satisfy some equivalence criterion.

: Up to an undetermined quantity which arises from constraint conditions.
} 
The starting time is arbitrary due to the fact that one can always write the representation of the state of a material point $\mathrm{X}$ at time $s$ as

$$
\mathscr{S}_{K}\left[\mathrm{r}_{\mathrm{K}}(\mathrm{X}), s\right]=\mathscr{S}_{\mathrm{K}}\left[\mathrm{r}_{\mathrm{x}}(\mathrm{X}), t_{\mathrm{s}}\right] \cup \mathscr{H}_{\mathrm{x}}\left[\mathrm{r}_{\mathrm{K}}(\mathrm{X}) ; t_{\mathrm{s}} \rightarrow s\right]
$$

for any given starting time, $t_{s}$, such that $t_{s} \in\left[t_{v}, s\right]$. This allows us to rewrite equation (3) with an explicit starting time as

$$
\sigma\left[r_{\kappa}(X), t\right]=\mathfrak{J}_{\kappa}\left\{\mathscr{S}_{\kappa}\left[r_{\kappa}(X), t_{s}\left[r_{\kappa}(X)\right]\right] ; F_{\kappa}\left[r_{\kappa}(X), s\right], T\left[r_{k}(X), s\right]\right\},
$$

where the same symbol $\mathfrak{I}_{x}$ is used to denote the new constitutive functional. As $\mathscr{S}_{k}\left[\boldsymbol{r}_{k}(\mathrm{X})\right.$, $t_{s}$ ] is a never-changing quantity in $\mathfrak{I}_{\kappa}$, we will suppress it in the notation and rewrite equation (S) as

$$
\sigma\left[r_{\kappa}(X), t\right]=\mathfrak{J}_{\kappa}\left\{F_{\kappa}\left[r_{\kappa}(X), \underset{s=t_{0}}{t} T\left[r_{k}(X), s\right]\right\},\right.
$$

where the same symbol $\mathfrak{J}_{x}$ denotes a new constitutive functional. We will use equation (6) in most of our work because of the explicit appearance of only the true variables in the representation of the response functional; it must be noted that the response functional in this equation has implicit in it a fixed unique initial state and also a fixed unique history of events going from the initial time to the starting time (i.e. the state at the starting time).

One restriction which is imposed on the constitutive functional is that of frame indifference. ${ }^{+}$This restriction allows us to write the constitutive equation (6) as

$$
\sigma\left[\mathbf{r}_{k}(\mathrm{X}), t\right]=\mathbf{R}_{\kappa}\left[\mathbf{r}_{\kappa}(\mathrm{X}), t\right] \mathfrak{J}_{\kappa}\left\{\mathbf{U}_{\kappa}\left[\mathbf{r}_{\kappa}(\mathrm{X}), s\right], T\left[\mathbf{r}_{k}(\mathrm{X}), s\right]\right\} \mathbf{R}_{\kappa}^{T}\left[\mathbf{r}_{\kappa}(\mathrm{X}), t\right],
$$

where $\mathbf{R}_{\kappa}\left[\mathbf{r}_{\kappa}(\mathrm{X}), s\right]$ and $\mathrm{U}_{\kappa}\left[\mathrm{r}_{\kappa}(\mathrm{X}), s\right]$ are the orthogonal and right symmetric parts of $F_{x}\left[r_{x}(X), s\right]$ in its polar decomposition.

An alternate form of equation (7) can be obtained by introducing a new functional $\mathscr{P}_{\kappa}$ such that

$$
\sigma\left[\mathrm{r}_{\kappa}(\mathrm{X}), t\right]=\mathrm{F}_{\kappa}(t) \mathscr{P}_{\kappa}\left\{\mathrm{C}_{\kappa}(\dot{s}), T(s)\right\} \mathrm{F}_{\kappa=t}^{T}(t)
$$

where reference to the particular material point $X$ is omitted from the notation and $\mathrm{C}_{\mathrm{x}}(s)=\mathrm{F}_{\alpha}^{T}(s) \mathrm{F}_{\kappa}(s)$. The functional $\mathscr{P}_{\kappa}$ is known as the functional for the second Piola-Kirchhoff stress tensor, and is defined in terms of the functional $\mathfrak{J}_{k}$ as

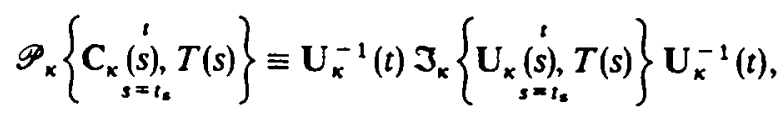

where $\mathrm{C}_{\mathrm{k}}(s)=\mathrm{U}_{\mathrm{k}}(s)^{2}$.

\section{A MATERIAL'S SYMMETRIES: DEFINITION IN A DETERMINISTIC PROCESS}

As an aid to understanding Material Symmetry (MS) and its relation to the state of a material point, let us consider the scheme in Fig. 2. The purpose of this scheme is to show the MS transformation $M$ which represents a symmetry of the material associated with its state at time $t_{2}$. For details of how a symmetry is represented by a linear transformation we refer the reader to [10]. The scheme shows the history of events the neighborhood (nbhd) of $X$ undergoes from an initial time, $t_{v}$, to some current time, $t$. In the scheme, $t_{1}, t_{2}$, and $t_{3}$ represent three intermediate points in time. $F_{1} \rightarrow F_{2} \rightarrow F_{3} \rightarrow F_{4}$ represent the actual history of deformations this nbhd undergoes from time $t_{v}$ to $t$. $F_{1} \rightarrow F_{2} \rightarrow M \rightarrow F_{3} \rightarrow F_{4}$ represents an alternate history of deformations. The two histories are the same up to an instantaneous reorganization of the nbhd at time $t_{2}$ (represented by the transformation $M$ in $F_{1} \rightarrow F_{2} \rightarrow M \rightarrow F_{3} \rightarrow F_{4}$ ).

\footnotetext{
See $[2-8,11]$ for more details.
} 


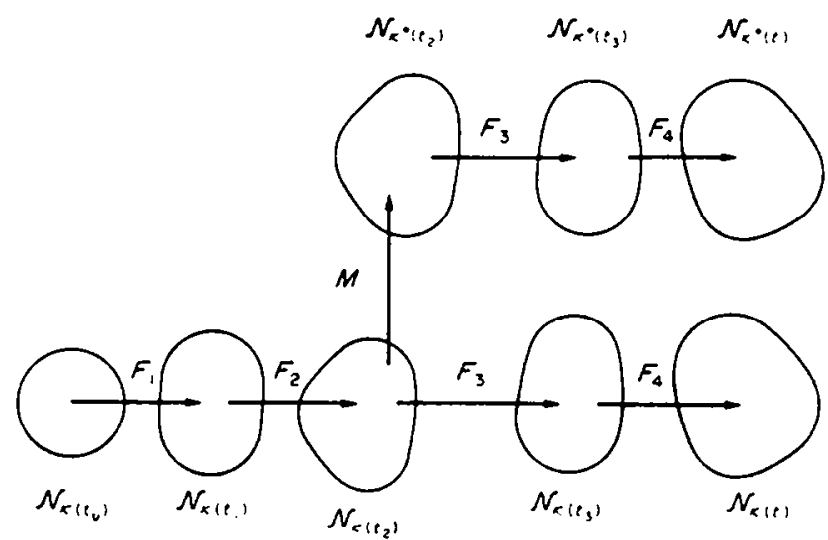

Fig. 2. Scheme showing two histories of events which differ only by an instantaneous reorganization at time $t_{2}$.

We will now present a definition of MS which expresses the notion of evolution in MS. If $M$ in Fig. 2 represents a symmetry of the material at its state at time $t_{2}$, the response of the material at any time $t>t_{2}$ should be identical for the two histories shown in the scheme, irrespective of what $F_{3} \rightarrow F_{4}$ is. In essence, one could subject the material at time $t_{2}$ to either $F_{3} \rightarrow F_{4}$ or $M \rightarrow F_{3} \rightarrow F_{4}$ without seeing any difference in the response of the material at time $t$. On the other hand, if we change the state of the material at time $t_{2}$ (by changing the history $\mathbf{F}_{1} \rightarrow \mathbf{F}_{2}$ ), then it is possible that the symmetries of the material at time $t_{2}$ will change, and $\mathbf{M}$ might no longer represent a symmetry of the material at time $t_{2}$. In other words, the symmetries of a material at any instant in time depends on the history of events which precede that instant.

\section{MATERIAL SYMMETRY GROUPS}

In Fig. 2, $M$ represented a MS expressed with respect to the configuration of the material at time $t_{2}, k\left(t_{2}\right)$. This transformation should map the space occupied by the nbhd of the particle in configuration $\kappa\left(t_{2}\right)$ onto itself. Therefore in the scheme, the history $\mathbf{M} \rightarrow \mathbf{F}_{3} \rightarrow \mathbf{F}_{4}$ should actually fall right on top of $F_{3} \rightarrow F_{4}$. As $M$ represents a symmetry of the material with respect to the configuration $\kappa\left(t_{2}\right)$, then $M$ will be a member of the mathematical group representing the MS of the particle at time $t_{2}$ and written with respect to the configuration $\kappa\left(t_{2}\right)$.

We will let $g_{\kappa}\left\{\mathscr{S}_{\kappa}\left[\mathrm{r}_{\kappa}(\mathrm{X}), s\right]\right\}$ denote the material symmetry group (MSG) of a material point at time $s$ represented with respect to configuration $\kappa$. As our notation and previous presentation suggests, we have assumed that MS depends on the state of the material. That is, MS in a material changes as we change the state of the material. To avoid any misunderstanding, we remind the reader that we have given a particular definition for the term "state". That is, when we say, "two nbhds of a material are in the same state", we mean that both nbhds have had the same initial state, and they have both undergone the same history of events. This particular use of the term "state" should not create any problems as long as it is not confused with the idea of "equivalent states". For more information we refer the reader to [11] and Section 2.

As mentioned in [10], Noll's rule will provide the means for obtaining the MSG of the material at time $s$ written with respect to any other configuration [given the MSG with respect to $\kappa(s)]$. That is,

$$
g_{\kappa(s)}\left\{\mathscr{S}_{\kappa}\left[\mathbf{r}_{\kappa}(\mathrm{X}), s\right]\right\}=\mathbf{F}_{\kappa}(s) g_{\kappa}\left\{\mathscr{S}_{\kappa}\left[\mathbf{r}_{\kappa}(\mathrm{X}), s\right]\right\} \mathbf{F}_{\kappa}(s)^{-1} \text {, }
$$

where $F_{\kappa}(s)$ is the deformation gradient comparing the nbhd of the particle at time $s$ to its nbhd in any configuration $\kappa$. In other words, we can represent the symmetries of the material at time $s$ in terms of any configuration of the body through an application of Noll's rule. 
As mentioned above, we consider a material's symmetries to depend on the state of the material point at the time under consideration. Traditionally (see $[2,4,7,9]$ ), this dependence has not been assumed. That is, a material is considered to have a single-nonchanging - MS. In the classical approach, when one speaks of a material's MS, one actually means its MS at the initial or starting state of the material. In either case, one could not talk about a change in MS as there would be no place in the definition which would allow MS to change for the material.

Our definition of MS allows us to avoid defining a material's symmetries through a particular, but otherwise arbitrary, state of the material. Letting MS be a function of the state of the material is a generalization of the classical idea of MS which not only reveals the arbitrary nature of the traditional definition, but also allows us to think of MS as a changing property of a material, and therefore allows us to speak of and follow the evolution of MS.

On the other hand, we must pay a price to achieve this generalization. This generalization requires that we mention the state of the material every time we speak of its MS. As this is usually inconvenient, we will sometimes use the following conventions. When we are speaking about a material's initial state, we will use the notation $g_{v x}(X)$ instead of $g_{k}\left[\mathscr{S}_{v k}(\mathrm{X})\right]$. When we are speaking about a predefined starting state of a constitutive equation - where there is no ambiguity about the starting state of the material-we will use the notation $g_{s x}(\mathrm{X})$ instead of $g_{x}\left[\mathscr{S}_{x}\left(\mathrm{X}, t_{\mathrm{s}}\right)\right]$. When there is a particular process (a history of events) in mind-where there is no ambiguity about the state of the material at each instant in the process-we will use the notation $g_{e x}(X, s)$ instead of $g_{k}\left[\mathscr{S}_{k}(X, s)\right]$.

We will call $g_{\mathrm{vx}}$ and $g_{\mathrm{sx}}$ the initial and starting MSGs respectively. As mentioned above, traditionally $g_{v \kappa}$ or $g_{s \kappa}$ would be taken as the material's absolute MSG. We will avoid using this traditional terminology and will always qualify our statements on MSGs by giving the state associated with the MS.

We call $g_{\text {ex }}(s)$ the evolving MSG at time $s$. This terminology is only meaningful if the reader knows what the state of the material is at time $s$. If there is any ambiguity about the state of the material at time $s$, this notation will not be meaningful, and therefore will only use this terminology when a predefined process is under consideration.

\section{FOLLOWING MATERIAL SYMMETRY IN A PROCESS}

In this section, we will set up the problem of following the evolution of a material's symmetries as we let the material undergo a known history of events. Up to this point, we have assumed the existence of a known measure of the response of the material. That is, in measuring for the existence of a MS, we have first assumed the existence of a quantity (or quantities) which identifies the response of the material. In our case, the "state" of Cauchy stress at the material point will be selected as the quantity which measures the response of a material to a history of events. We will call the response of a material point identical or indistinguishable for two histories of events if the corresponding histories of the Cauchy stress tensor are the same. In essence, from now on, we will consider the Cauchy stress tensor as the final measure of the response of a material to a history of events. ${ }^{\ddagger}$

We now will look at how MS can be calculated if we are given the constitutive functional for the stress response of the material. As mentioned in Section 2, the stress response of a material can be given as

$$
\boldsymbol{\sigma}(t)=\mathfrak{J}_{\kappa}\left\{\mathbf{F}_{\kappa} \stackrel{i}{(s)}, T(s)\right\}
$$

For reasons which will shortly become obvious, we will rewrite this equation as

$$
\sigma(t)=\mathfrak{J}_{\alpha}\left\{F_{\alpha}(s), T(s) ; F_{x=t_{s}}\left(\underset{\alpha=t_{2}}{t}, T(\alpha)\right\}\right.
$$

\footnotetext{
'In practice, in the traditional approach, the selection of the starting state dictates the material's symmetry even though the definition gives the false impression that MS is a unique property of the material.

Depending on the theory under consideration, one might consider the heat fux vector or any other quantity (or combination of quantities) as the measure of the response of the material.
} 
where the range of $s$ and $\alpha$ are given as $\left(t_{3}, t_{2}\right]$ and $\left(t_{2}, t\right]$ respectively. There is no approximation implied in rewriting (11) in the form (12).

The ideas discussed in Section 3 will now be formulated in the following definition.

Definition. If $\mathbf{H}$ is a member of $g_{\kappa}\left[\mathscr{S}_{\kappa}\left(\mathrm{X}, t_{2}\right)\right]$, then the material's symmetry at time $t_{2}$ requires that

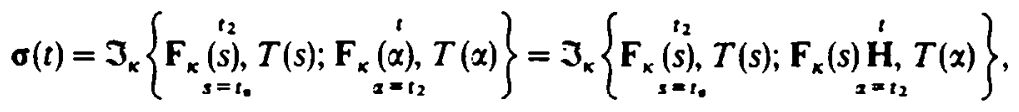

for every possible history of events $\mathscr{H}_{k}\left(t_{2} \rightarrow t\right)$ and for every arbitrary time $t$. Alternately, if we can find a transformation $H$ which satisfies (13) for every history of events $\mathscr{H}_{N}\left(t_{2} \rightarrow t\right)$ and for every arbitrary time $t$, then $\mathbf{H}$ will be a member of $g_{k}\left[\mathscr{S}_{k}\left(X, t_{2}\right)\right]$; that is, $\mathbf{H}$ will be a member of the MSG of the material point with respect to the given state at time $t_{2}$. In this definition, $\mathbf{H}$ is related to $\mathbf{M}$ of Fig. 2 by an application of Noll's rule.

We can now state the problem of following the evolution of a material's symmetries as the material undergoes a known history of events.

Problem. Given $g_{\kappa}\left[\mathscr{S}_{\kappa}\left(\mathrm{X}, t_{1}\right)\right]$, and given a history $\mathscr{H}_{\kappa}\left(t_{1} \rightarrow t_{2}\right)$, find every $\mathbf{H}$ such that

$$
\left.\sigma(t)=\mathfrak{J}_{\kappa}\left\{\mathbf{F}_{\kappa}(s), T(s) ; \mathbf{F}_{\kappa}(\alpha), T(\alpha)\right\}=t_{\alpha=t_{2}}^{t}\right\} \mathfrak{J}_{\kappa}\left\{\mathbf{F}_{\kappa}(s), \underset{s=t_{1}}{t_{2}}, T(s) ; \mathbf{F}_{\kappa}(\alpha) \underset{\alpha=t_{2}}{\mathbf{H}}, T(\alpha)\right\}
$$

for every history $\mathscr{H}_{\alpha}\left(t_{2} \rightarrow t\right)$, and for every arbitrary time $t$. That is, given the MSG at time $t_{1}$ and the history of events from time $t_{1}$ to $t_{2}$, find the MSG at time $t_{2}$. It is worth mentioning that, as the starting time can be taken as any arbitrary time, in (14) we have replaced the starting time by time $t_{1}$.

There is not enough structure in the functional $\mathfrak{J}_{x}$ to solve the restriction given by (14) for any $\mathbf{H}$ other than obtaining the trivial solution $\mathbf{H}=\mathbf{I}$. Imposing the restriction of frameindifference on the functional gives the functional sufficient structure to evaluate nontrivial solutions for $\mathbf{H}$ in (14).

Recalling (8), if we impose the constraint of frame indifference on the response functional of the Cauchy stress, we will have

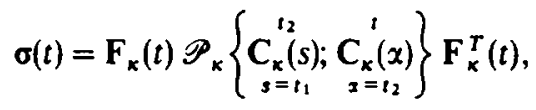

where $\mathscr{P}_{\kappa}$ was the functional for the second Piola-Kirchhoff stress tensor, $\mathbf{C}_{\kappa}(s)=$ $F_{\kappa}^{T}(s) F_{\kappa}(s)$, and the dependence of $\mathscr{P}_{\kappa}$ on temperature is suppressed in the notation. Using (15) to represent the stress response of the material, our general problem can be restated as follows.

Problem. Given $g_{\kappa}\left[\mathscr{S}_{\kappa}\left(\mathrm{X}, t_{1}\right)\right]$, and given a history $\mathscr{H}_{\kappa}\left(t_{1} \rightarrow t_{2}\right)$, find every $\mathbf{H}$ such that,

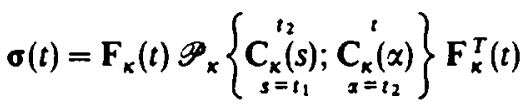

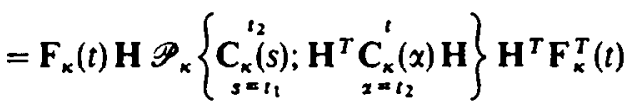

for every arbitrary history $\mathscr{H}_{\kappa}\left(t_{2} \rightarrow t\right)$, and for every arbitrary time $t$.

Solution. As, for every $H \in g_{\kappa}\left[\mathscr{S}_{\kappa}\left(X, t_{1}\right)\right]$, we know that

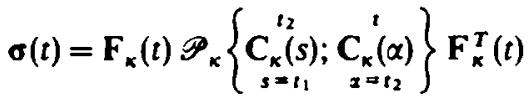

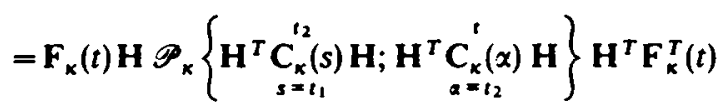

is true, a comparison of (16) and (17) gives the solution of the problem as follows:

$$
g_{\kappa}\left[\mathscr{S}_{\kappa}\left(\mathrm{X}, t_{2}\right)\right] \supseteq g_{\kappa}\left[\mathscr{S}_{\kappa}\left(\mathrm{X}, t_{1}\right)\right] \cap g_{\mathrm{d}_{\kappa}}\left(t_{1} \rightarrow t_{2}\right)
$$

where

$$
g_{\mathrm{d}_{\kappa}}\left(t_{1} \rightarrow t_{2}\right)=\left\{\mathbf{H} \mid \mathbf{H}^{T} \mathbf{C}_{\kappa}(s) \mathbf{H}=\mathbf{C}_{\kappa}(s) \quad \text { for every } \quad s \in\left(t_{1}, t_{2}\right]\right\}
$$


We will call $g_{\mathrm{d}_{\mathrm{x}}}\left(t_{1} \rightarrow t_{2}\right)$ the deformation's symmetry group (DSG) from $t_{1}$ to $t_{2}$. It is easy to show that $g_{d_{x}}$ is a group and that DSGs have the same properties as the MSGs with respect to changes in the reference configuration $\kappa$. That is, all properties mentioned in [10] about MSGs will equally apply to DSGs $\left(g_{\mathrm{d}_{\mathrm{x}^{*}}}=\overline{\mathbf{F}} g_{\mathrm{d}_{\mathrm{k}}} \overline{\mathbf{F}}^{-1}\right)$.

The problem of following a material's symmetries has reduced, in part, to intersecting the deformation's symmetries (DSs) with the material's initial symmetries. There is no guarantee that by going through this process we will find all the symmetries of a particular material. That is, even though we are guaranteed that every MS identified by this method will be a member of the final MS, there might be certain symmetries which are not identified by this procedure. In particular, as we have not assumed any particular form or structure for the response functional $\mathscr{P}_{\kappa}$, there might be certain symmetries which are a result of the characteristics of the particular material under consideration and which we will not obtain by the procedure presented above. The preceding comments are the reason for not using an equality in (18).

Relation (18) identifies the symmetries common to all simple materials which initially have a MSG of $g_{\kappa}\left[\mathscr{S}_{\kappa}\left(\mathrm{X}, t_{1}\right)\right]$ at time $t_{1}$ and subsequently undergo a given history of events from time $t_{1}$ to time $t_{2}$. Each particular simple material will most possibly have more symmetries in its MSG at time $t_{2}$ than identified by the intersection of $g_{k}\left[\mathscr{S}_{k}\left(\mathrm{X}, t_{1}\right)\right]$ and $g_{\mathrm{d}_{\mathrm{K}}}\left(t_{1} \rightarrow t_{2}\right)$. The identification of the symmetries which come from a material's particular characteristics will not be done here. The cases of a particular model of inelasticity and a particular model of solid polymer crystallization will be published elsewhere.

We will proceed in the next section to follow the evolution of MS for materials whose group of MSGs at time $t_{1}$ can be generated from an orthogonal generator (see [10] for definition).

\section{MATERIALS WHICH INITIALLY HAVE ORTHOGONAL GENERATORS}

In this section we will look at the evolution of MS for those materials whose group of MSGs at time $t_{1}$ can be generated from orthogonal generators, i.e. there is some configuration $\kappa$ such that $g_{\kappa}\left[\mathscr{S}_{\kappa}\left(\mathrm{X}, t_{1}\right)\right]$ is a subgroup of the full group of orthogonal transformations. For these materials, we will consider the evolution of MS for particular histories of deformations. In each case, the procedure to evaluate the material's final MS will be to intersect the material's initial MSG with the DSG associated with the particular deformations under consideration.

As in the previous section, $t_{1}$ will denote the time for which we are given the MSG of the material and $t_{2}$ is the time at which we desire to know the material's MSG. As we will only consider states of the material at time $t_{1}$ for which the group of MSGs at time $t_{1}$ can be generated from an orthogonal generator, we can select the configuration $\kappa$ such that $g_{\kappa}\left[\mathscr{S}_{\kappa}\left(\mathrm{X}, t_{1}\right)\right] \subset \mathcal{O}$, i.e. all the MSG's members are orthogonal transformations. For such a $g_{k}\left[\mathscr{S}_{k}\left(X, t_{1}\right)\right]$, which only contains orthogonal members, it is obvious that in calculating $g_{k}\left[\mathscr{S}_{k}\left(\mathrm{X}, t_{1}\right)\right] \cap g_{\mathrm{d}_{k}}\left(t_{1} \rightarrow t_{2}\right)$, we need only evaluate the orthogonal members of $g_{\mathrm{d}_{\mathrm{k}}}\left(t_{1} \rightarrow t_{2}\right)$. That is, even though there might be non-orthogonal members in $g_{\mathrm{d}_{x}}\left(t_{1} \rightarrow t_{2}\right)$, only orthogonal members of $g_{\mathrm{d}_{k}}\left(t_{1} \rightarrow t_{2}\right)$ will be in the intersection of $g_{\mathrm{d}_{k}}\left(t_{1} \rightarrow t_{2}\right)$ and $g_{\kappa}\left[\mathscr{S}_{\kappa}\left(\mathrm{X}, t_{1}\right)\right]$.

An important point to note is that, once we have found $g_{x}\left[\mathscr{S}_{k}\left(\mathrm{X}, t_{1}\right)\right] \cap g_{\mathrm{d}_{x}}\left(t_{1} \rightarrow t_{2}\right)$ for a configuration $\kappa$ in which $g_{\kappa}\left[\mathscr{S}_{\kappa}\left(\mathrm{X}, t_{1}\right)\right] \subset \mathcal{O}$, then $g_{\kappa^{\prime}}\left[\mathscr{S}_{\kappa}\left(\mathrm{X}, t_{1}\right)\right] \cap g_{\mathrm{d}_{\kappa^{\prime}}}\left(t_{1} \rightarrow t_{2}\right)$ for any arbitrary $\kappa^{\prime}$ can be found by applying Noll's rule to $g_{\kappa}\left[\mathscr{S}_{\kappa}\left(\mathrm{X}, t_{1}\right)\right] \cap g_{\mathrm{d}_{\kappa}}\left(t_{1} \rightarrow t_{2}\right) .^{+}$Therefore, the selection of $\kappa$ such that $g_{k}\left[\mathscr{S}_{k}\left(\mathrm{X}, t_{1}\right)\right] \subset \mathcal{O}$ does not introduce any restrictions on the results which will be presented, but this selection will simplify the presentation.

In the following sections, we will separately look at the evolution of initially isotropic, transversely isotropic, and orthotropic materials for certain classes of deformation. As we will use the anisotropic tensors in the later developments, a short presentation of the subject follows.

\section{ANISOTROPIC TENSORS}

In obtaining the results presented later, we have used the results of Rivlin and Smith [12] on anisotropic tensors. The following is a short presentation on anisotropic tensors. The

\footnotetext{
'This follows from the fact that $g_{k}=\overline{\mathbf{F}} g_{k} \overline{\mathbf{F}}^{-1}$ and $g_{\mathrm{d}_{k}}=\overline{\mathbf{F}} g_{\mathrm{d}_{\alpha}} \overline{\mathbf{F}}^{-1}$ for $\overline{\mathbf{F}}$ given as the deformation gradient comparing the configuration of the nbhd of the material point in $\kappa^{\prime}$ with its configuration in $\kappa$.
} 
scope of this presentation is restricted to its particular use in this paper. A more detailed presentation can be found in $[12,13]$.

Smith and Rivlin [12] have shown that for a given group $g$ of orthogonal transformations, there is a set of anisotropic tensors $\alpha_{i_{1}}^{(p)} \ldots i_{m}$, for $p=1,2, \ldots$, with the property

$$
\alpha_{i_{1}}^{(p)} \ldots i_{m}=H_{i_{1} j_{1}} \ldots H_{i_{m} j_{m}} \alpha_{j_{1} \ldots j_{m}}^{(p)},
$$

for every $H_{i j} \in g$ (the standard summation convention is assumed on repeated indices). This set forms a basis for the construction of any tensor $G_{i_{1} \ldots i_{n}}$ that has the property,

$$
G_{i_{1}} \ldots i_{n}=H_{i_{1} j_{1}} \ldots H_{i_{n} j_{n}} G_{j_{1}} \ldots j_{n} \text {, }
$$

for every $H_{i j} \in g$. That is, every $\mathrm{G}$ which satisfies (21) for all $H_{i j} \in g$ can be written as

$$
\mathrm{G}=b_{1} \boldsymbol{\beta}^{(1)}+\ldots+b_{q} \boldsymbol{\beta}^{(q)} \text {, }
$$

for some scalars $b_{1}, \ldots, b_{q}$ and tensors $\boldsymbol{\beta}^{(1)}, \ldots, \boldsymbol{\beta}^{(q)}$ where each tensor $\boldsymbol{\beta}^{(i)}$ is either an anisotropic tensor of $g$ or the tensor product of anisotropic tensors of $g$. This implies that any $\mathbf{G}$ which can be written in the form (22) will satisfy (21) and vice versa.

As we will only be concerned with the case where $G$ is a second-rank tensor, equation (21) can be written as

$$
\mathbf{G}=\mathbf{H G H} \mathbf{H}^{T} \text {. }
$$

We will confine the rest of this presentation to such a $\mathbf{G}$.

What follows is a presentation of triclinic, monoclinic, orthotropic, and transversely isotropic materials. For each case we first define an orthogonal generator and then provide the anisotropic tensors associated with this generator. As we only consider those $\mathbf{G}$ which are second-order symmetric tensors in a three-dimensional space, we will only present the anisotropic tensors relevant to this case (see [12] for more details).

(1) Triclinic material: A material is considered triclinic-pinacoidal if its group of MSGs can be generated from $D_{t r i}= \pm\{I\}$, where $I$ is the identity. That is, the material is only indifferent to a central inversion. There are nine anisotropic tensors associated with $D_{\mathrm{tr}}$ and they are given as

$$
\alpha_{i j}^{(11)}=\delta_{i 1} \delta_{j 1}, \alpha_{i j}^{(12)}=\delta_{i 1} \delta_{j 2}, \ldots, \alpha_{i j}^{(33)}=\delta_{i 3} \delta_{j 3},
$$

where $\delta_{i j}$ is the Kronecker delta. All simple materials which satisfy the constraint of frame indifference are at least triclinic.

(2) Monoclinic material: A material is considered monoclinic-prismatic if its group of MSGs can be generated from $D_{\text {mon }}= \pm\left\{\mathbf{I}, \mathbf{R}_{1}\right\}$, where $\mathbf{R}_{\mathbf{1}}=\operatorname{Diag}(-1,1,1)$. That is, the material is indifferent to central inversions and reflections about one plane of symmetry (in at least one configuration of the body). There are five anisotropic tensors associated with $D_{\text {mon }}$ and they are given as $\alpha^{(11)}, \alpha^{(22)}, \alpha^{(33)}, \alpha^{(23)}$, and $\alpha^{(32)}$, where $\alpha^{(1)}$ is defined in (24).

(3) Orthotropic (rhombic) material: A material is considered orthotropic or rhombic-dipyramidal if its group of MSGs can be generated from $D_{\text {orth }}= \pm\left\{\mathbf{I}, \mathbf{R}_{1}, \mathbf{R}_{2}, \mathbf{R}_{3}\right\}$, where $I$ and $\mathbf{R}_{1}$ are the same as given above, and $\mathbf{R}_{2}=\operatorname{Diag}(1,-1,1)$, and $\mathbf{R}_{3}=\operatorname{Diag}(1,1$, -1 ). That is, the material is indifferent to central inversion and reflections about three perpendicular planes of symmetry (in at least one configuration of the body). There are three anisotropic tensors associated with $D_{\text {orth }}$ and they are given as $\alpha^{(11)}, \alpha^{(22)}$, and $\alpha^{(33)}$.

(4) Transversely isotropic material: A material is considered transversely isotropic if its group of MSGs can be generated from $D_{\text {tran }}= \pm\left\{\mathbf{I}, \mathbf{R}_{1}, \mathbf{M}_{\omega}, \mathbf{R}_{1} \mathbf{M}_{\omega} ;\right.$ for all $\left.\omega\right\}$, where $I$ and $\mathbf{R}_{1}$ are given above, and

$$
\mathbf{M}_{\omega}=\left(\begin{array}{ccc}
\cos (\omega) & \sin (\omega) & 0 \\
-\sin (\omega) & \cos (\omega) & 0 \\
0 & 0 & 1
\end{array}\right)
$$

for $0 \leq \omega<2 \pi$. That is, the material is indifferent to central inversions, reflections about any plane which passes through a preferred axis of the material, and reflections about a plane perpendicular to the preferred axis of the material (in at least one configuration of the body). There are two anisotropic tensors associated with $D_{\text {tran }}$ and they are given as $\alpha^{(33)}$, and $\alpha^{(11)}+\alpha^{(22)}$. 


\section{INITIALLY ISOTROPIC MATERIALS}

For initially isotropic materials $g_{k}\left[\mathscr{S}_{x}\left(\mathrm{X}, t_{1}\right)\right]$ is the full group of orthogonal transformations; that is, $g_{\kappa}\left[\mathscr{S}_{\kappa}\left(\mathrm{X}, t_{1}\right)\right]=\mathcal{O}$. Therefore, the current MSG of the material will satisfy

$$
g_{\kappa}\left[\mathscr{S}_{\kappa}\left(\mathrm{X}, t_{2}\right)\right] \supseteq \mathcal{O} \cap g_{\mathrm{d}_{\kappa}}\left(t_{1} \rightarrow t_{2}\right) \text {. }
$$

We note that it may be possible for $g_{k}\left[\mathscr{S}_{\kappa}\left(\mathrm{X}, t_{2}\right)\right]$ to contain orthogonal and unimodular nonorthogonal transformations not contained in $\mathcal{O} \cap g_{\mathrm{d}_{k}}\left(t_{1} \rightarrow t_{2}\right)$. What follows is the evaluation of $\mathcal{C} \cap g_{\mathrm{d}_{\mathrm{x}}}\left(t_{1} \rightarrow t_{2}\right)$ for particular deformation histories.

\subsection{Equal triaxial extension}

For the case where the deformation history from $t_{1}$ to $t_{2}$ only contains equal triaxial extensions,

$$
\mathrm{C}_{\mathrm{x}}(s)=a(s) \mathrm{I},
$$

where $a(s)$ is an arbitrary function of $s \in\left(t_{1}, t_{2}\right]$, and $I$ is the identity. Such a $C_{k}(s)$ is forminvariant to all orthogonal transformations, and therefore $g_{d_{x}}=\mathcal{C}$. As $g_{d_{k}} \cap \mathcal{O}=\mathcal{O}$, therefore,

$$
g_{\kappa}\left[\mathscr{S}_{\kappa}\left(\mathrm{X}, t_{2}\right)\right] \supseteq \mathcal{O} \text {. }
$$

That is, any initially isotropic material which is only subjected to a history of uniform triaxial extensions will remain isotropic.

\subsection{Uniaxial (equal biaxial) extensions}

Let us consider the case where the deformation history from $t_{1}$ to $t_{2}$ only contains uniaxial extensions along a fixed axis of the material;

$$
\mathrm{C}_{\mathrm{k}}(s)=\mathrm{Q}\left[a_{1}(s) \boldsymbol{\alpha}^{(33)}+a_{2}(s)\left(\boldsymbol{\alpha}^{(11)}+\alpha^{(22)}\right)\right] \mathrm{Q}^{T},
$$

where $a_{1}(s)$ and $a_{2}(s)$ are arbitrary functions of $s \in\left(t_{1}, t_{2}\right]$, where $Q$ is a constant orthogonal linear transformation, and where $\alpha^{(33)}$ and $\alpha^{(11)}+\alpha^{(22)}$ are the anisotropic tensors associated with the group $D_{\text {tran }}$. Such a history of $C_{k}(s)$ is form-invariant to all transformations in $\mathbf{Q} D_{\text {tran }} \mathbf{Q}^{T}$. Because, as long as $a_{1}(s) \neq a_{2}(s)$ for some $s \in\left(t_{1}, t_{2}\right], \mathbf{Q} D_{\text {tran }} \mathbf{Q}^{T}$ will contain all the orthogonal transformations which are in $g_{\mathrm{d}_{\kappa}}$, then $g_{\mathrm{d}_{\kappa}} \cap \mathcal{O}=\mathbf{Q} D_{\text {tran }} \mathbf{Q}^{T}$ and

$$
g_{x}\left[\mathscr{S}_{*}\left(\mathrm{X}, t_{2}\right)\right] \supseteq \mathbf{Q} D_{\text {tran }} \mathbf{Q}^{T} \text {. }
$$

That is, any initially isotropic material which is only subjected to uniaxial extensions along a fixed axis of the material will at least be a transversely isotropic material after the completion of the deformations. The direction of the preferred axis of transverse isotropy is determined by $\mathbf{Q}$.

\subsection{Unequal triaxial extensions}

Consider the case where the deformation history from $t_{1}$ to $t_{2}$ only contains triaxial extensions along a set of three fixed orthogonal axes of the material,

$$
\mathrm{C}_{\kappa}(s)=\mathrm{Q}\left[a_{1}(s) \alpha^{(11)}+a_{2}(s) \alpha^{(22)}+a_{3}(s) \alpha^{(33)}\right] \mathrm{Q}^{T},
$$

where $a_{1}(s), a_{2}(s)$, and $a_{3}(s)$ are arbitrary functions of $s \in\left(t_{1}, t_{2}\right]$, where $\mathbf{Q}$ is a constant orthogonal linear transformation, and where $\alpha^{(11)}, \alpha^{(22)}$, and $\alpha^{(33)}$ are the anisotropic tensors associated with the group $D_{\text {ortb }}$. Such a history of $C_{x}(s)$ is form-invariant to all transformations in $Q D_{\text {orth }} Q^{T}$. Because, as long as $a_{1}(s) \neq a_{2}(s) \neq a_{3}(s) \neq a_{1}(s)$ for some $s \in\left(t_{1}, t_{2}\right], Q D_{\text {orth }} Q^{r}$ will contain all the orthogonal transformations which are in $g_{\mathrm{d}_{k}}$, then $g_{\mathrm{d}_{\boldsymbol{k}}} \cap \mathcal{O}=\mathbf{Q} D_{\text {orth }} \mathbf{Q}^{T}$ and

$$
g_{\kappa}\left[\mathscr{S}_{\kappa}\left(\mathrm{X}, t_{2}\right)\right] \supseteq \mathbf{Q} D_{\text {orth }} \mathbf{Q}^{T}
$$

That is, any initially isotropic material which is only subjected to triaxial extensions along a fixed set of orthogonal axes of the material will at least be an orthotropic material after the completion of the deformations. The preferred axes of orthotropy are given by $\mathbf{Q}$. 


\subsection{Combined simple shear and triaxial extensions}

For the case where the deformation history from $t_{1}$ to $t_{2}$ is a combination of triaxial extensions along a fixed set of orthogonal axes of the material with simple shear in one plane of the triaxial extensions,

$$
\mathrm{C}_{\mathrm{k}}(s)=\mathrm{Q}\left[a_{1}(s) \alpha^{(11)}+a_{2}(s) \alpha^{(22)}+a_{3}(s) \alpha^{(33)}+a_{4}(s)\left(\alpha^{(23)}+\alpha^{(32)}\right)\right] \mathbf{Q}^{T},
$$

where $a_{1}(s), \ldots, a_{4}(s)$ are arbitrary functions of $s \in\left(t_{1}, t_{2}\right]$, where $Q$ is a constant orthogonal linear transformation, and where $\alpha^{(11)}, \alpha^{(22)}, \alpha^{(33)}, \alpha^{(23)}+\alpha^{(32)}$ are the anisotropic tensors associated with $D_{\text {mon }}$. For such a deformation history, it is simple to show that

$$
g_{k}\left[\mathscr{S}_{\mathrm{K}}\left(\mathrm{X}, t_{2}\right)\right] \supseteq \mathrm{Q} D_{\text {mon }} \mathrm{Q}^{T} \text {. }
$$

That is, the final material will at least be invariant to central inversions and a reflection about the plane of simple shear.

\section{INITIALLY TRANSVERSELY ISOTROPIC MATERIAL}

For an initially transversely isotropic material $g_{\kappa}\left[\mathscr{S}_{\kappa}\left(\mathrm{X}, t_{1}\right)\right]=\mathbf{P} D_{\mathrm{tran}} \mathbf{P}^{T}$ for some constant orthogonal transformation $\mathbf{P}$ and the group $D_{\text {tran }}$. We will select the coordinate axes such that $\mathbf{P}=\mathbf{I}$. This selection introduces no particular restrictions, but it simplifies the presentation. Therefore, we will consider a material for which $g_{\alpha}\left[\mathscr{S}_{\alpha}\left(X, t_{1}\right)\right]=D_{\text {tran }}$; that is, its preferred axis of transverse isotropy is along the third coordinate axis. The current MSG of the material will satisfy

$$
g_{\mathrm{x}}\left[\mathscr{S}_{\mathrm{x}}\left(\mathrm{X}, t_{2}\right)\right] \supseteq D_{\mathrm{tran}} \cap g_{\mathrm{d}_{\mathrm{x}}}\left(t_{1} \rightarrow t_{2}\right) .
$$

What follows is the calculation of $D_{\operatorname{tran}} \cap g_{\mathrm{d}_{\mathrm{k}}}\left(t_{1} \rightarrow t_{2}\right)$ for several particular deformation histories. As we have already evaluated $\mathcal{O} \cap g_{\mathrm{d}_{\mathrm{x}}}\left(t_{1} \rightarrow t_{2}\right)$ for these histories in the last section, and as $D_{\text {tran }} \cap g_{\mathrm{d}_{x}}\left(t_{1} \rightarrow t_{2}\right)=D_{\text {tran }} \cap\left[\mathcal{O} \cap g_{\mathrm{d}_{x}}\left(t_{1} \rightarrow t_{2}\right)\right]$, we can use the results of the last section.

The proof of the results presented here are primarily by inspection and are not presented in detail. Presentation of the proofs are quite lengthy and are, to a great extent, parallel to those in $[10,11]$. Therefore, the reader is referred to $[10,11]$ for more details.

\subsection{Equal triaxial extensions}

For this history of deformations, we have shown that, $\mathcal{C} \cap g_{\mathrm{d}_{\mathrm{x}}}\left(t_{1} \rightarrow t_{2}\right)=\mathcal{C}$ and therefore

$$
g_{\kappa}\left[\mathscr{S}_{x}\left(\mathrm{X}, t_{2}\right)\right] \supseteq D_{\text {tran }} .
$$

Therefore, after a history only involving equal triaxial extensions, an initially transversely isotropic material will remain at least transversely isotropic.

\subsection{Uniaxial (equal-biaxial) extensions}

For this history of deformations we have shown that $\mathcal{O} \cap g_{\mathrm{d}_{x}}\left(t_{1} \rightarrow t_{2}\right)=\mathbf{Q} D_{\operatorname{tran}} \mathbf{Q}^{T}$ and therefore

$$
g_{x}\left[\mathscr{S}_{\times}\left(\mathrm{X}, t_{2}\right)\right] \supseteq D_{\operatorname{tran}} \cap \mathbf{Q} D_{\operatorname{tran}} \mathbf{Q}^{T}
$$

There are three distinct cases:

(1) If the two preferred axes of transverse isotropy (those associated with $D_{\text {tran }}$ and $\mathbf{Q} D_{\operatorname{tran}} \mathbf{Q}^{T}$ ) become aligned, then $\mathbf{Q} D_{\operatorname{tran}} \mathbf{Q}^{T}=D_{\operatorname{tran}}$ and therefore

$$
g_{k}\left[\mathscr{S}_{\kappa}\left(\mathrm{X}, t_{2}\right)\right] \supseteq D_{\text {tran }} \text {. }
$$

(2) If the preferred axes of transverse isotropy of $D_{\operatorname{tran}}$ and $Q D_{\operatorname{tran}} \mathbf{Q}^{T}$ are perpendicular, then $D_{\text {tran }} \cap \mathbf{Q} D_{\text {tran }} \mathbf{Q}^{T}=\mathbf{M}_{\phi} D_{\text {orth }} \mathbf{M}_{\phi}^{T}$ for some $\phi$, and

$$
\mathbf{M}_{\phi}=\left(\begin{array}{ccc}
\cos (\phi) & \sin (\phi) & 0 \\
-\sin (\phi) & \cos (\phi) & 0 \\
0 & 0 & 1
\end{array}\right) \text {. }
$$


Therefore,

$$
g_{\kappa}\left[\mathscr{S}_{\kappa}\left(\mathrm{X}, t_{2}\right)\right] \supseteq \mathbf{M}_{\phi} D_{\text {orth }} \mathbf{M}_{\phi}^{T}
$$

(3) If neither of the above is true (i.e. the preferred axes of $D_{\text {tran }}$ and $Q D_{\text {tran }} Q^{T}$ are neither parallel nor perpendicular), then $D_{\text {tran }} \cap Q D_{\text {tran }} \mathbf{Q}^{T}=\mathbf{M}_{\phi} D_{\text {mon }} \mathbf{M}_{\phi}^{T}$ for some $\phi$, and $\mathbf{M}_{\phi}$ given as above.

Therefore, depending on the axis of uniaxial extension (given by $\mathbf{Q}$ ), the final material will be transversely isotropic, orthotropic, or monoclinic.

\subsection{Non-equal triaxial extensions}

For this history of deformations, we have shown that $\mathcal{C} \cap g_{\mathrm{d}_{x}}\left(t_{1} \rightarrow t_{2}\right)=Q D_{\text {orth }} \mathbf{Q}^{T}$ and therefore

$$
g_{\kappa}\left[\mathscr{S}_{\kappa}\left(\mathrm{X}, t_{2}\right)\right] \supseteq D_{\mathrm{Iran}} \cap \mathbf{Q} D_{\text {orth }} \mathbf{Q}^{r}
$$

There are three distinct cases:

(1) In the case where the preferred axis of transverse isotropy (represented by the term $D_{\text {tran }}$ ) becomes aligned with one of the directions of orthotropy (represented by the term $\mathbf{Q} D_{\text {orth }} \mathbf{Q}^{T}$ ) we will have

$$
g_{\kappa}\left[\mathscr{S}_{\kappa}\left(\mathrm{X}, t_{2}\right)\right] \supseteq \mathbf{Q} D_{\text {orth }} \mathbf{Q}^{T}
$$

(2) If the preferred axis of transverse isotropy is in one of the planes of symmetry associated with $Q D_{\text {orih }} \mathbf{Q}^{T}$ and not along any of its axes, then

$$
g_{k}\left[\mathscr{S}_{k}\left(\mathrm{X}, t_{2}\right)\right] \supseteq \pm \mathbf{Q}\left\{\mathbf{I}, \mathbf{R}_{1}\right\} \mathbf{Q}^{T}
$$

or

$$
g_{\kappa}\left[\mathscr{S}_{\kappa}\left(\mathrm{X}, t_{2}\right)\right] \supseteq \pm \mathbf{Q}\left\{\mathbf{I}, \mathbf{R}_{2}\right\} \mathbf{Q}^{T}
$$

or

$$
g_{\kappa}\left[\mathscr{S}_{\kappa}\left(\mathrm{X}, t_{2}\right)\right] \supseteq \pm \mathbf{Q}\left\{\mathbf{I}, \mathbf{R}_{3}\right\} \mathbf{Q}^{T}
$$

depending on which plane of symmetry of $Q D_{\text {orth }} Q^{T}$ it falls in.

(3) If neither of the above is true, then

$$
g_{\kappa}\left[\mathscr{P}_{\kappa}\left(\mathrm{X}, t_{2}\right)\right] \geq D_{\mathrm{tri}}
$$

Therefore, depending on the axes of the triaxial extensions (which are given by $\mathbf{Q}$ ), the final material will at least be orthotropic, monoclinic, or triclinic.

\section{INITIALLY ORTHOTROPIC MATERIAL}

For an initially orthotropic material $g_{\kappa}\left[\mathscr{S}_{\kappa}\left(\mathrm{X}, t_{1}\right)\right]=\mathbf{P} D_{\text {orth }} \mathbf{P}^{T}$ for some orthogonal $\mathbf{P}$. As in the last section, we will select the coordinate axes such that $\mathbf{P}=\mathbf{I}$, without any loss in generality. Therefore, the current MSG of the material will be

$$
g_{k}\left[\mathscr{S}_{\kappa}\left(\mathrm{X}, t_{2}\right)\right] \supseteq D_{\text {orth }} \cap g_{d_{k}}\left(t_{1} \rightarrow t_{2}\right)
$$

What follows is the calculation of $D_{\text {orth }} \cap g_{\mathrm{d}_{\mathrm{k}}}\left(t_{1} \rightarrow t_{2}\right)$ for several deformation histories. As $D_{\text {orth }} \cap g_{\mathrm{d}_{k}}\left(t_{1} \rightarrow t_{2}\right)=D_{\text {orth }} \cap\left[O \cap g_{\mathrm{d}_{k}}\left(t_{1} \rightarrow t_{2}\right)\right]$, and we have already calculated $O \cap g_{d_{k}}\left(t_{1} \rightarrow t_{2}\right)$ in the section on initially isotropic material, we will use the results and notation of that section.

Most of the proofs in this section are by inspection and will not be presented in detail. Similar presentations were done in $[10,11]$, to which we direct the reader for more details.

\subsection{Equal triaxial extensions}

For this history of deformations, we have shown that, $\mathcal{O} \cap g_{d_{x}}\left(t_{1} \rightarrow t_{2}\right)=\mathcal{O}$ and therefore

$$
g_{\kappa}\left[\mathscr{S}_{\kappa}\left(\mathrm{X}, t_{2}\right)\right] \supseteq D_{\text {orth }} \text {. }
$$

Therefore, after a history only involving equal triaxial extension, an initially orthotropic material will remain at least orthotropic. 
10.2. Uniaxial (equal-biaxial) extensions

For this history of deformations, we have shown that $\mathcal{O} \cap g_{d_{k}}\left(t_{1} \rightarrow t_{2}\right)=Q D_{\operatorname{tran}} Q^{T}$ and therefore

$$
g_{k}\left[\mathscr{S}_{\mathrm{K}}\left(\mathrm{X}, t_{2}\right)\right] \supseteq D_{\mathrm{orth}} \cap \mathbf{Q} D_{\mathrm{tran}} \mathbf{Q}^{T}
$$

There are three distinct cases:

(1) If the preferred axis of $Q D_{\text {tran }} Q^{T}$ is along one of the preferred axes of $D_{\text {orth }}$, then

$$
g_{k}\left[\mathscr{S}_{k}\left(\mathrm{X}, t_{2}\right)\right] \supseteq D_{\text {orth }} \text {. }
$$

(2) If the preferred axis of $Q D_{\operatorname{tran}} Q^{T}$ is in one of the planes of symmetry of $D_{\text {orth }}$ but not along any of its axes, then

$$
g_{\kappa}\left[\mathscr{S}_{\kappa}\left(\mathrm{X}, t_{2}\right)\right] \supseteq \pm\left\{\mathbf{I}, \mathbf{R}_{1}\right\}
$$

or

$$
g_{\kappa}\left[\mathscr{S}_{\kappa}\left(\mathbf{X}, t_{2}\right)\right] \supseteq \pm\left\{\mathbf{I}, \mathbf{R}_{2}\right\}
$$

or

$$
g_{k}\left[\mathscr{S}_{k}\left(\mathrm{X}, t_{2}\right)\right] \supseteq \pm\left\{\mathbf{I}, \mathbf{R}_{3}\right\}
$$

depending on which plane of $D_{\text {orth }}$ it falls in.

(3) If neither of the above is true, then

$$
g_{\mathrm{x}}\left[\mathscr{S}_{\mathrm{x}}\left(\mathrm{X}, t_{2}\right)\right] \supseteq D_{\mathrm{tri}}
$$

Therefore, depending on the axis of uniaxial extension (given by $\mathbf{Q}$ ), the final material will be orthotropic, monoclinic, or triclinic.

10.3. Non-equal triaxial extensions

For this history of deformations, we have shown that, $O \cap g_{\mathrm{d}_{x}}\left(t_{1} \rightarrow t_{2}\right)=\mathrm{Q} D_{\text {orth }} \mathrm{Q}^{T}$ and therefore

$$
g_{\kappa}\left[\mathscr{I}_{\kappa}\left(\mathrm{X}, t_{2}\right)\right] \supseteq D_{\text {orth }} \cap \mathbf{Q} D_{\text {orth }} \mathbf{Q}^{T}
$$

There are three distinct cases:

(1) If all three axes of orthotropy coincide, then

$$
g_{x}\left[\mathscr{S}_{\kappa}\left(\mathrm{X}, t_{2}\right)\right] \supseteq D_{\text {orth }} \text {. }
$$

(2) If only one of the preferred axes of $D_{\text {orth }}$ and $Q D_{\text {orth }} Q^{T}$ coincide, then

$$
g_{\kappa}\left[\mathscr{S}_{\kappa}\left(\mathbf{X}, t_{2}\right)\right] \supseteq \pm\left\{\mathbf{I}, \mathbf{R}_{\mathrm{t}}\right\}
$$

or

$$
g_{x}\left[\mathscr{S}_{*}\left(X, t_{2}\right)\right] \supseteq \pm\left\{\mathbf{I}, \mathbf{R}_{2}\right\}
$$

or

$$
g_{\kappa}\left[\mathscr{S}_{\kappa}\left(\mathrm{X}, t_{2}\right)\right] \supseteq \pm\left\{\mathbf{I}, \mathbf{R}_{3}\right\}
$$

depending on which axes coincide.

(3) If neither of the above is true, then

$$
g_{\kappa}\left[\mathscr{S}_{\kappa}\left(\mathrm{X}, t_{2}\right)\right] \supseteq D_{\mathrm{tri}} \text {. }
$$

Therefore, depending on the axes of the triaxial extension (which are given by $Q$ ), the final material will at least be orthotropic, monoclinic, or triclinic.

\section{CONCLUDING REMARKS}

There are several points we would like to emphasize. First, to obtain these results, we have not assumed any structure, other than that imposed by frame indifference, on the stress response functional of the material. That is, if frame indifference is a reasonable restriction for all simple materials, then these results must be true for all simple materials.

Second, in each case, we have evaluated the minimum symmetry the material must have after the particular deformation; that is, there might be symmetries the material has gained 
due to its particular structure which are not considered in this paper. Therefore, this minimum symmetry the material will finally have is common to all simple materials as it is purely due to the deformations the material has undergone.

Third, as will be shown in later publications on inelasticity and polymer crystallization, once one imposes more structure on the constitutive functional, it is possible to predict with greater accuracy the evolution of symmetries.

Finally, we would like to mention that there might be several reasons for a material not to have the minimum symmetries predicted here. First, the material might not be a simple material. Second, it might be going through a non-continuum transition. That is, the events occurring on a molecular level cannot be followed by only measuring the evolution of continuum variables. Third, as an MS is an absolute property, small changes in the parameters of a problem can totally change the MS. That is, for example, a material cannot be both isotropic and orthotropic (it must be one or the other), and therefore a small change in the parameters of the problem might make a material which was predicted to become isotropic finally become orthotropic (or to have any other acceptable MS). The analysis of how sensitive a material's properties are to changes in the parameters influencing their change is a separate issue, which must be addressed separately for each particular material.

\section{REFERENCES}

1. J. L. Erickson, On the symmetry and stability of thermoelastic solids. J. appl. Mech. 45,740 (1978).

2. C. Truesdell, The Elements of Continuum Mechanics. Springer-Verlag, Heidelberg (1985).

3. A. J. M. Spencer, Continuum Mechanics. Longman, London (1980).

4. A. C. Eringen, Continuum Phy'sics. Academic Press. New York (1975).

5. D. C. Leigh, Non-linear Continuum Mechanics. McGraw-Hill. New York (1968).

6. C. Truesdell and W. Noll. The Non-linear Field Theories of Mechunics, Vol. III;3. (Edited by S. Flugge). Springer-Verlag. Heidelberg (1965).

7. C. Truesdell, A first Course in Rational Continuum Mechanics, Vol. I. General Concepts. Academic Press, New York (1977).

8. W. Noll, A mathematical theory of the mechanical behavior of continuous media. Archs Rational Mech. Anal. 2. 197 (1958).

9. C. Truesdell, Six Lectures on Modern .Vatural Philosophy. Springer-Verlag, Heidelberg (1966).

10. M. Negahban and A. S. Wineman, Material symmetry and the evolution of anisotropies in a simple materialI. Change of reference configurations. Int. J. Non-linear Mech. (in press).

11. M. Negahban, Material symmetry and the evolution of anisotropies in first gradient theories of material behavior, inelasticity; polymer crystallization. Dissertation, University of Michigan (1988).

12. G. F. Smith and R. S. Rivlin. The anisotropic tensors. Q. appl. Math. XV, 308 (1957).

13. A. J. M. Spencer. Theory of invariants. In Continuum Physics, Vol. I. (Edited by A. C. Eringen). Academic Press, New York (1975). 